\title{
The history of mathematical modeling in hydrocephalus
}

\author{
Michelle J. Clarke, M.D., and Fredric B. Meyer, M.D. \\ Department of Neurologic Surgery, Mayo Clinic, Rochester, Minnesota
}

\begin{abstract}
$\checkmark$ The mathematical modeling of hydrocephalus is a relatively young field. The discipline evolved from Hakim's initial description of the brain as a water-filled sponge. Nagashima and colleagues subsequently translated this description into a computer-driven model by defining five important system rules. A number of researchers have since criticized and refined the method, providing additional system constraints or alternative approaches. Such efforts have led to an increased understanding of ventricular shape change and the development of periventricular lucency on imaging studies. However, severe limitations exist, precluding the use of the mathematical model to influence the operative decisions of practicing surgeons. In this paper, the authors explore the history, limitations, and future of the mathematical model of hydrocephalus.
\end{abstract}

\section{KEY WORDS - hydrocephalus • mathematical model • finite element analysis • pressure-volume relationship}

$\mathrm{H}$ YDROCEPHALUS, a complex series of diseases, involves the interaction of multiple organs (brain, CSF, and blood) inside a semienclosed space (the skull). The fundamental change seen in hydrocephalic symptoms is a perturbation of ICP or the volume of intracranial contents. Often, such alteration is accompanied by changes in ventricular size, the degree of change being limited by the volume of material in the cranial vault and by intrinsic brain properties (elasticity, fluid flow, and so forth).

The Monro-Kellie hypothesis simplifies the problem by underscoring the interaction of intracranial contents and the fixed volume of the intracranial vault. Unfortunately, this approach does not take into account the properties of the individual organs, specifically, the material properties of the brain itself. It is these material properties that potentially dictate the severity and permanence of neurological compromise in the face of hydrocephalic changes. In addition, time is not represented by the Monro-Kellie hypothesis, and yet the rapidity of hydrocephalic changes and the effect of time on the material properties of the brain may influence the severity and chronicity of physiological and neurological changes.

In the early 1970s, Hakim ${ }^{8}$ and colleagues ${ }^{9}$ began the process of delineating the physical properties of the brain that lead to, and result from, the changes of hydrocephalus. Nagashima and colleagues ${ }^{14}$ translated and further refined these ideas into an FEM that simulated the changes

Abbreviations used in this paper: $\mathrm{CSF}=$ cerebrospinal fluid; ICP = intracranial pressure; FEM = finite element model. of acute ventricular outlet obstruction. This 2D model remains the foundation on which most recent computer simulations are based. Although mathematical models continue to improve, a number of current limitations must be overcome to increase their utility.

\section{OUTLINE OF MODEL REQUIREMENTS}

A hydrocephalic model must conform to basic physical laws while reproducing the physiological changes associated with the disease. Although simple scenarios, including piston models and balloons, have been used to describe the complexities of the pressure-volume changes that occur in hydrocephalus, they do not adequately describe the interaction between the ventricular area and ICP.

Hakim $^{8}$ attempted a more complete mechanical model by describing brain parenchyma as "an open cell sponge made of viscoelastic material." These cells were thought to be of various sizes and filled with liquid, including venous blood or extracellular fluid. Hakim believed that because these cells were indirectly connected to the atmosphere through the CSF/venous system, these cells would allow the brain/fluid system to compress. Essentially, although the liquid itself is incompressible, fluid could be "wrung out" of the brain and cranial vault through the venous sinuses, allowing the theoretically incompressible brain tissue to effectively collapse. Thus, Hakim $^{8}$ and his associates ${ }^{9}$ attributed parenchymal deformation to the progressive collapse of parenchymal veins, which he believed formed the large "cells of the sponge." 
The sponge model allows one to imagine that the force expanding the ventricles is resisted by parenchymal structures and the CSF/venous system. Hakim correctly assumed that the increased ventricular pressure, although transmitted to the parenchyma, dissipated with distance from the ventricles, resulting in a pressure gradient or "effective CSF pressure." In addition, hydraulic resistance to the transfer of CSF to the venous sinus system provided a pressure gradient from the ventricles, the location of CSF production, to the parenchymal venous system (specifically the superior sagittal sinus), the location of CSF absorption. ${ }^{8,9}$

Unfortunately, Hakim was limited in attempting to model his system mathematically. He relied on estimating the stress-strain relationship directly by assuming that the brain was a homogeneous viscoelastic substance. In addition, he was limited in describing the complex shape of the brain and ventricles and relied on a hollow sphere model.

\section{BASIC PRINCIPLES OF MATHEMATICAL MODELING}

Whereas Hakim provided a framework of needs, Nagashima et al. ${ }^{14}$ translated it into a mathematical system. Nagashima and colleagues relied heavily on Biot, who outlined the linear mechanical properties of a poroelastic medium. Biot used the Hooke law of linear elasticity, the Darcy law of the fluid flow through a porous medium, and the Navier-Stokes equations describing the motion of fluids to describe mathematically the poroelastic substance and form the Biot consolidation theory. ${ }^{2}$ In 1987 Nagashima and associates ${ }^{14}$ reinterpreted Biot's concepts and outlined four principles of the hydrocephalic biomechanical model system: the law of conservation of mass, the Darcy law, the Hooke law, and the Terzaghi effective stress principle.

The first principle is rooted in the law of conservation of mass, originally formulated in 1789 by Lavoisier. ${ }^{4}$ Two important components of this principle relate to hydrocephalus: first, there is a fixed mass of solid brain matter (including, among other components, neurons, glia, and connective tissue); and second, there is a fixed mass of interstitial fluid.

Conservation of momentum, another basic tenet of physics, provides the second principle. ${ }^{4}$ Conservation of momentum involving fluid through a porous medium is known as the Darcy law. ${ }^{5}$ In conjunction with the law of conservation of mass, these principles were originally used to describe the flow of fluid through soil or an aquifer. In hydrocephalus, these principles are used to describe the flow of interstitial fluid. Essentially, like most biological tissues, the brain is described as biphasic: a solid state containing cellular structures and extracellular matrix and a liquid state containing water. Masses in both states must be conserved. This description is the mathematical interpretation of fluid in Hakim's sponge-like parenchymal model.

Like a sponge, the brain has the ability to deform under an applied load. The Hooke law of elasticity, the third principle, models deformation as a spring. Essentially, the amount of deformation (strain) is directly proportional to the load applied (stress), modified by the elastic modulus (or the Young modulus), an inherent property of the material. ${ }^{4}$ Like many materials, the brain has a range of elastic behaviors over which this law remains valid. As with a spring, the brain can be deformed by a force and return to its original shape when the load is removed.

The Hooke law deals with the deformation of solid objects; given the porous nature of the brain, the law must be modified extensively, becoming the fourth principal. The most effective solution is provided by the study of soil mechanics, akin to modeling loose particles acting as a unit. In the case of the brain, the solid structures act as soil particles, and extracellular fluid takes up the air space that would normally be found between these particles. Given that this fluid is noncompressible, it exerts a force on the solid components of the brain, known as the "pore water pressure." The calculated stress required to cause displacement, otherwise known as the Terzaghi effective stress principle, is simply the difference between total stress and pore water pressure. ${ }^{26}$

Armed with a mathematical representation of the biomechanics of the poroelastic medium based on these four principles, Nagashima and colleagues were able to use the FEM to mimic the system on a computer. Simply put, an object (in this case the parenchymal/CSF system) can be divided into small pieces (finite elements) to which material properties can be defined. Boundary conditions (such as the ventricular-parenchymal interface or the fixed skull) are also assigned. Finally, a time-dependent loading condition can be applied to the system, which allows the theoretical change of each finite element to be discerned.

Nagashima and associates' ${ }^{14}$ model correlated well with clinical examples of hydrocephalus. They used singleslice computed tomography scans to create a 2D FEM of the brain. In this model, they stipulated six material properties for each element (for example, elasticity and fluid permeability), which differed between white and gray matter. Extracellular fluid was modeled as pure water. Important boundary conditions for extracellular fluid included a pressure of $20 \mathrm{~mm} \mathrm{Hg}$ at the ventricular wall and $10 \mathrm{~mm} \mathrm{Hg}$ at the brain surface, providing a hydrostatic pressure gradient. The model was run in 20 time steps, calculating interstitial stress, pressure, and ventricular shape at each time point.

\section{VENTRICULAR SHAPE CHANGES}

Nagashima and colleagues ${ }^{14}$ noted that the greatest stress concentrations and largest deformations occurred at the anterolateral angle of the frontal horn followed by the occipital and temporal horns, similar to conditions in clinical cases of acute obstructive hydrocephalus. In addition, with each progressive time step, the interstitial pressure increase extended further into the periventricular region. These areas of increased pressure correspond to regions of fluid accumulation or edema in clinical cases.

Pena and associates ${ }^{17,18}$ further refined the linear poroelastic FEM, creating a time-dependent demonstration of ventricular expansion. In this model, increased ventricular pressure resulted in "inflation" of the frontal and occipital horns and lateral flattening of the thalami. Through this model, these authors were able to assess areas of compression and expansion, thus providing the most accurate representation of the stress concentrations and ventricular 
anatomy. In addition, their model also promoted a greater understanding of the extracellular fluid shifts leading to edema and periventricular lucency.

\section{PERIVENTRICULAR LUCENCY AND EXTRACELLULAR FLUID CHANGES}

Periventricular lucency, a hydrocephalic change first noted by Naidich et al. ${ }^{15}$ is usually seen as a blurring of the lateral margins of the frontal horns of the lateral ventricles on computed tomography scans. ${ }^{1}$ This phenomenon is theorized to result from disruption of the ependymal surface, an assertion supported by experimental results..$^{13}$ It is thought that ventricular dilation leads to wall breakdown, allowing CSF extravasation and decompression of the ventricles.

Pena and colleagues' ${ }^{17}$ model of ventricular dilation correctly predicts the location of periventricular lucency through "voids." The "void ratio" is defined as the volume of the extracellular space (voids) divided by the volume of the matrix. These voids are the "holes" in Hakim's sponge and the areas in which extracellular fluid collects. More fluid can collect in areas of void expansion, compared with areas of void compression. The model not only demonstrates intraparenchymal stresses but also provides insight into edema accumulation. Areas of highest ventricular concavity correspond to the ventricular horns. In fact, Pena et al. theorized that the concavity itself concentrates expansive stresses on the area, opening the voids and causing fluid accumulation.

Perhaps more interesting, these effective expansive stresses damage the ependymal lining itself by stretching and tearing. Theoretical ependymal permeability increases in these areas allow still more fluid accumulation. In addition, the decrease in structural integrity results in greater ventricular dilation, permitting still more ependymal damage. However, this theory cannot be tested in the linear elastic model. An elastoplastic model, which accounts for permanent changes, would be required.

Authors of further studies have defined the parenchymal-CSF interface by defining strict parameters for the ependymal lining. Kaczmerek and colleagues ${ }^{10}$ defined this boundary condition as stiffer and less permeable than white matter. Given the minimal thickness of the ependyma, this area is modeled as an elastic membrane. Theoretically, the permeability of the ependyma is increased because of damage caused by ventricular dilation. This model includes a dilation-dependent ependymal permeability function for the small-strain case. However, the method is limited by estimates of the Young modulus and ependymal stiffness, given the absence of experimental data, and by an inability to model the variability of ependymal permeability in large deformation analysis.

Despite these limitations, this model clearly demonstrates solid matrix dilation near the ventricle, effectively providing a physical model of periventricular edema. In addition, the model reveals greater compaction of gray matter, consistent with clinical and experimental data that edema is predominantly located in the white matter. ${ }^{21,27}$

\section{MODELING THE CSF SPACES}

Compartmental analysis of the ventricular spaces com- bined with animal experimentation provides a different approach to mathematical modeling of ICP and hydrocephalus compared with the methods outlined earlier. Compartmental systems explore the nonlinear compliance of the CSF system and its relationship with pressure, shifting the focus from the mechanical properties of the brain itself. In these compartmental models, computer simulations can be more easily correlated with pressure-volume measurements derived from intraventricular CSF infusions or extractions in animal models.

Marmarou and colleagues ${ }^{11,12}$ pioneered this direction of study, hypothesizing that the major parameters in ICP dynamics are compliance and resistance to fluid absorption. Compliance measures the distensibility of a compartment such as a ventricle and is defined as the slope of the pressure-volume curve of that compartment. Resistance to fluid absorption occurs primarily at the arachnoid granulations. For absorption to occur, the pressure differential between the subarachnoid space and venous system must be overcome. The modeling system of Marmarou et al. ${ }^{12}$ abstracts the CSF system as an equivalent electrical circuit: a "generator" is represented by the formation of a constant "current" of CSF; a nonlinear "capacitor," by CSF storage; and a "resistor," by the area of CSF absorption.

This model correctly predicted the results of a continuous fluid infusion, bolus fluid infusion, and bolus fluid extraction in an experimental cat model. ${ }^{11}$ Normally, infused intraventricular volume causes an abrupt rise in ICP, followed by a slow return to equilibrium. Eventually, volume can exceed physiological compensation, resulting in an inability to completely reequilibrate or a continued increase in pressure. This result was seen in both bolus and continuous infusions. However, decreased ICP following bolus extraction reduces, eliminates, or reverses the pressure gradient required for CSF absorption at the arachnoid granulations. Intracranial pressure initially normalizes through the formation of new CSF until the pressure gradient is reestablished and absorption recommences. Two conclusions resulted from this model and the corresponding animal studies: the pressure-volume curve is a fundamental system property, and there is a time-dependent pattern of pressure change with volume perturbation. ${ }^{11,12}$

Expanding on the electrical circuit model, Rekate explored the interaction among all four ventricular compartments as well as the resistance of the communications between compartments. ${ }^{19,20}$ In cases of ICP perturbations with unaffected communication between compartments, the CSF system acts as a single compartment, with the only resistance occurring at the arachnoid granulations absorption site. However, in special cases such as the development of resistance between compartments, the utility of modeling independent compartments is apparent. For instance, a colloid cyst at the foramen of Monro can lead to increased flow resistance between the lateral and third ventricles, resulting in univentricular dilation. In addition, data in this study emphasize the importance of venous resistance in the creation of hydrocephalus. Increased venous pressure subsequently increases absorption resistance by increasing the subarachnoid-venous system pressure differential, ultimately resulting in CSF accumulation and hydrocephalus.

Combining both the CSF- and brain-based models, Spertell ${ }^{22}$ considered the brain as a single-compartment 
viscoelastic spherical shell. Using this system, the brain response to ventricular pulse pressure and the importance of the subarachnoid space can be determined. Increased ventricular pressure is transmitted to the subarachnoid space where the subarachnoid pressure slowly decays to baseline. In the case of hydrocephalus, in which long-duration pressure waves are common, pressure decay in the subarachnoid space occurs faster than that in the ventricle, and outward ventricular displacement is favored. Importantly, the mathematical equations used in the model may be solved for the specific viscoelastic properties of the parenchyma, including elastic constants. Although the single-compartment spherical model is not directly translatable into the animal model, it demonstrates the potential of compartment-based models providing testable estimates of parenchymal parameters.

Modeling CSF compartments provides understanding of ventricular volume regulation and is more easily correlated with animal studies than is modeling based on stressstrain principles in solid intracranial structures. Given that each CSF space contains liquid, pressure throughout the space is homogeneous; thus, pressure is easily determined before, during, and after insertion or removal of a known volume of fluid. Unlike solid-state models in which parenchymal heterogeneity and fluid saturation result in complicated functions, the CSF compartment model can be simplified with the time-dependent viscoelastic compliance function. However, the interaction between the CSF compartment and parenchymal changes must both be modeled for a true hydrocephalus model.

\section{PARAMETER DEFINITIONS}

One of the most frustrating aspects in the mathematical study of hydrocephalus is the lack of realistic estimates of the mechanical properties of the living human brain. In fact, Nagashima and colleagues' ${ }^{14}$ original consolidation model has been shown to be severely flawed based on incorrect parameter assumptions. Drake and colleagues ${ }^{6}$ and Tenti and associates ${ }^{24}$ focused on the Poisson ratio (a measure of compressibility) and demonstrated the inherently unreliable results that would occur. In this case, Nagashima and colleagues modeled the porous matrix as essentially incompressible, as is the extracellular fluid (modeled as water). Obviously, this approach makes it impossible to truly model changes in brain configuration. Indeed, as Pena et al. ${ }^{17}$ have stated, "only simultaneous precise measurements of all of these parameters (pressure gradient, ventricular distention, and tissue stiffness) would clarify this issue." This issue becomes readily apparent when one considers the problems in estimating the elastic modulus.

Taylor and Miller ${ }^{23}$ demonstrated the historical difficulty in parameter estimation using a 2D FEM to calculate the Young modulus (elastic modulus). As these researchers noted, given the ability of a biphasic material to redistribute, the Young modulus will appear to change in a loadand rate-dependent manner. To explain this in Hakim's terms, a sponge filled with water may transiently support an initial load; but as the water is squeezed out over time (redistribution of extracellular fluid), the load is progressively supported only by the sponge, and deformation ensues. Numerically, the effect is impressive. Whereas pre- vious estimates of the Young modulus have ranged from 3000 to $10,000 \mathrm{~Pa}$, Taylor and Miller calculated a value of $584.4 \mathrm{~Pa}$, a marked reduction in material stiffness.

Although accurate material property measurements are paramount to producing an appropriate model of hydrocephalus, a simple value is unlikely to be the answer. As Taylor and Miller hypothesized, material properties of elastic tissue will likely change with time and the applied load. Furthermore, "the history of strain affects the stress ... there is considerable difference in stress response between loading and unloading." 7

Pang and Altschuler ${ }^{16}$ elaborated on this variation in their study of low-pressure hydrocephalus, introducing the concept of ventricular hysteresis. Creating hypothetical pressure-volume curves, these authors explained the effect of alterations in parenchymal elasticity and the necessity of restoring the original elasticity to improve treatment outcome. Essentially, ventricular dilation results in alterations in the viscoelastic modulus, as theorized by Taylor and Miller. ${ }^{23}$ However, Pang and Altschuler ${ }^{16}$ note that on treatment (such as inserting a shunt), the brain does not necessarily revert to the predisease state, and therefore the pressure-volume relationship in the brain is different during ventricular contraction.

In addition, the brain cannot be assumed to exist only in the elastic state. There is a point at which the speed and degree of load combine to cause permanent nonelastic (or plastic) changes. In other words, the brain is "overstretched" and irreparably altered. This fact had hypothetical importance in a previous study of very low pressure hydrocephalus. ${ }^{3}$ In that case, the speed and degree of change in ventricular size likely caused permanent stretching and tearing of the descending neural pathways. To further this theory, modeling permanent changes may become important, given the often repetitive stresses inherent in patients who cycle through repeated shunt failures. Thus, a true "elastoplastic" model combining both types of deformation is required.

Rekate $^{19,20}$ has demonstrated the importance of a model system that can be correlated and refined by animal model systems. Although the CSF compartment model does not address actual parenchymal mechanical property changes leading to alterations in compliance, it does suggest the degree and direction of perturbation resulting from these changes. The ease of correlation allows iterative refinement of the mathematical modeling through correlation with animal studies. This important step, often lacking in purely theoretical parenchyma-based models, is essential to adjust and validate the mathematical model. In addition, proof that single-bolus fluid injections accurately estimate parenchymal compliance can be clinically useful in determining compliance changes in patients, rendering a diagnosis, planning treatment, and predicting outcome.

\section{CURRENT LIMITATIONS AND FUTURE RESEARCH}

Although the mathematical modeling of hydrocephalus has progressed well beyond the Monro-Kellie compartments and Hakim's brain sponge, clinically applicable results have been disappointing. A truly relevant model must not only lead to greater understanding of the disease, but also allow researchers to test clinically relevant sce- 
narios accurately. In their review, Tenti et al. ${ }^{25}$ noted two major future challenges.

First, a "nonlinear constitutive [stress-strain] equation" 25 must be found that is relevant for all clinical hydrocephalic conditions. The "small-strain" limitations are not valid over the true range of the disease. Even with perfectly determined material property definitions, the equations are inherently unreliable. Currently, most calculations are based on small-strain estimates wherein the deformation is limited to a few percentage points. Beyond this point, the large strain model requires one of two approaches: a geometrically nonlinear model using a full nonlinear strain tensor, or an estimation using computational mechanics. Most researchers have pursued the second method whereby the time-dependent load is divided into a number of small steps, each modeled as a small-strain estimate. Akin to elementary calculus, increasing the number of small steps will result in convergence on the true value. Expanding a system used to describe infinitesimal changes to encompass the macroscopic alterations of hydrocephalus will not produce a globally applicable system.

Second, appropriate boundary conditions must be imposed, ${ }^{25}$ including not only the material properties of the organs themselves, but also the initial conditions of the system (such as ventricular and intraparenchymal pressure). In this case, clinical experiments, basic science advances, and theoretical approaches must combine to continue the pursuit of these properties.

\section{CONCLUSIONS}

An adequate understanding of hydrocephalic diseases involves the interaction of four major research arms. Basic science provides physical parameters of organ properties and an understanding of the disease on a cellular level. Computer-based mathematical modeling provides a macroscopic biomechanical framework. Animal studies allow further refinements of the mathematical models and a platform on which theoretical interventions can be tested. Finally, clinical correlation is required to ensure the accuracy of the model systems.

Mathematical modeling of hydrocephalus combines basic physics and materials science with a refined abstraction of brain properties. Serious limitations in the utility of these models exist, and progress in eliminating them has been slow. However, further refinements can provide a useful platform for learning about the disease as well as a testbed for future treatments.

\section{References}

1. Alp MS: Periventricular lucency on computed tomography associated with hydrocephalus: what is the cause? Surg Neurol 44:285-286, 1995

2. Biot MA: General theory of three dimensional consolidation. J Appl Phys 12:1244-1258, 1941

3. Clarke MJ, Maher CO, Nothdurft G, Meyer F: Very low pressure hydrocephalus. Report of two cases. J Neurosurg 105: 475-478, 2006

4. Cutnell JD, Johnson KW: Physics, ed 2. Hoboken, NJ: John Wiley \& Sons, 1992

5. Darcy H: Appendix D, in The Public Fountains of the City of Dijon, (English Transl). Paris: Bookseller of the Imperial Corps of Bridges, Highways and Mines, 1856

6. Drake JM, Mostachfi O, Tenti G, Sivaloganathan S: Realistic simple mathematical model of brain biomechanics for computer simulation of hydrocephalus and other brain abnormalities. Can J Neurol Sci 23 (1 Suppl): S33, 1996 (Abstract)

7. Fung YC: Biomechanics. Mechanical Properties of Living Tissues, ed 2. New York: Springer Science + Business Media, 1993

8. Hakim S: Biomechanics of hydrocephalus. Acta Neurol Latinom 1 (1 Suppl): 169-194, 1971

9. Hakim S, Venegas JG, Burton JD: The physics of the cranial cavity, hydrocephalus and normal pressure hydrocephalus: mechanical interpretation and mathematical model. Surg Neurol 5: 187-210, 1976

10. Kaczmerek M, Subramaniam RP, Neff SR: The hydromechanics of hydrocephalus: steady-state solutions for cylindrical geometry. Bull Math Biol 59:295-323, 1997

11. Marmarou A, Shulman K, LaMorgese J: Compartmental analysis of compliance and outflow resistance of the cerebrospinal fluid system. J Neurosurg 43:523-534, 1975

12. Marmarou A, Shulman K, Rosende RM: A nonlinear analysis of the cerebrospinal fluid system and intracranial pressure dynamics. J Neurosurg 48:332-344, 1978

13. Milhorat TH, Clark RG, Hammock MK, McGrath PP: Structural, ultrastructural, and permeability changes in the ependyma and surrounding brain favoring equilibration in progressive hydrocephalus. Arch Neurol 22:397-407, 1970

14. Nagashima T, Tamaki N, Matsumoto S, Horwitz B, Seguchi Y: Biomechanics of hydrocephalus: a new theoretical model. Neurosurgery 21:898-903, 1987

15. Naidich TP, Epstein F, Lin JP Kricheff II, Hochwald GM: Evaluation of pediatric hydrocephalus by computed tomography. Radiology 119:337-345, 1976

16. Pang D, Altshuler E: Low-pressure hydrocephalic state and viscoelastic alterations in the brain. Neurosurgery 35:643-655, 1994

17. Pena A, Bolton MD, Whitehouse H, Pickard JD: Effects of brain ventricular shape on periventricular biomechanics: a finite element analysis. Neurosurgery 45:107-118, 1999

18. Pena A, Harris NG, Bolton MD, Czosnyka M, Pickard JD: Communicating hydrocephalus: the biomechanics of progressive ventricular enlargement revisited. Acta Neurochir Suppl 81: 59-63, 2002

19. Rekate HL: Circuit diagram of the circulation of cerebrospinal fluid. Pediatr Neurosurg 21:248-253, 1994

20. Rekate HL: The usefulness of mathematical modeling in hydrocephalus research. Childs Nerv Syst 10:13-18, 1994

21. Reulen HJ, Graham R, Spatz M, Klatzo I: Role of pressure gradients and bulk flow in dynamics of vasogenic brain edema. J Neurosurg 46:24-35, 1977

22. Spertell RB: The response of brain to transient elevations in intraventricular pressure. J Neurol Sci 48:343-352, 1980

23. Taylor Z, Miller K: Reassessment of brain elasticity for analysis of biomechanisms of hydrocephalus. J Biomech 37:1263-1269, 2004

24. Tenti G, Drake JM, Sivaloganathan S: Brain biomechanics: mathematical modeling of hydrocephalus. Neurol Res 22:19-24, 2000

25. Tenti G, Sivaloganathan S, Drake JM: Brain biomechanics: steady-state consolidation theory of hydrocephalus. Can Appl Math Quart 7:93-110, 1999

26. Terzaghi K, Peck RB, Mesri G (eds): Soil Mechanics in Engineering Practice, ed 3. New York: John Wiley \& Sons, 1996, pp 71-208, 223-236

27. Weller RO, Mitchell J: Cerebrospinal fluid edema and its sequelae in hydrocephalus. Adv Neurol 28:111-23, 1980

Manuscript submitted January 15, 2007.

Accepted March 2, 2007.

Address reprint requests to: Michelle J. Clarke, M.D., Department of Neurologic Surgery, Mayo Clinic, 200 First Street SW, Rochester, Minnesota 55905. email: Clarke.michelle@mayo.edu. 